\title{
Development of New Muon Monitors for J-PARC Neutrino Experiment
}

\author{
Kenji Yasutome*t \\ Kyoto University \\ E-mail: vasutome.kenji.38rest.kyoto-u.ac.jp
}

J-PARC neutrino experiments use the proton beam to produce intense $v_{\mu}$ beam. One of the motivations of the experiment is to discover the CP violation in the lepton sector. The J-PARC muon monitor (MUMON) is one of the monitors and the only one to monitor the secondary beam bunch-by-bunch, therefore MUMON is essential for the beam operation. The experiment is planning to increase the proton beam power in the near future. Current MUMON detectors do not have high radiation tolerance. We need to develop new detectors for future high intensity beam. Candidates are diamond detectors, PMT (Photon Multiplier Tube) and EMT (Electron Multiplier Tube). According to data so far, EMT is the best candidate. Initial test results are shown and the next beam test plan is introduced in this talk.

The 21st international workshop on neutrinos from accelerators (NuFact2019)

August 26 - August 31, 2019

Daegu, Korea

\footnotetext{
* Speaker.

${ }^{\dagger}$ on behalf of T2K Neutrino Beam Group
} 


\section{J-PARC neutrino experiment}

J-PARC neutrino experiments use the proton beam to generate intense $v_{\mu}$ beam towards Super Kamiokande (SK) which is located $295 \mathrm{~km}$ away from J-PARC at 2.5-degree off-axis. The beamline can produce either a $v$ or $\bar{v}$ beam with $1 \% v_{e}$ contamination and a peak energy of about 0.6 $\mathrm{GeV}$. One of the physics motivations of $\mathrm{T} 2 \mathrm{~K}$ is to measure neutrino oscillation parameters, notably $\delta_{C P}$.

\section{J-PARC muon monitor (MUMON)}

MUMON is located on-axis about $100 \mathrm{~m}$ away from neutrino target and monitor the direction and intensity of muons from pion, kaon decays to understand the $v$ beam profiles. MUMON is one of the monitors used in the experiment, the only one to monitor the secondary beam bunch-bybunch, therefore it is essential for the beam operation. Two types of detectors are used, an array of Silicon PIN photodiodes (Si) and Ion Chamber (IC). Each array has $7 \times 7$ channels and covers $150 \mathrm{~cm}^{2}$. These detectors have shown the required performance since the experiment commissioning in 2009. We found some issues with the current detectors, shown in Figure $\mathbf{l}$. A signal of the Si sensors decrease $1 \%$ per $5 \times 10^{20}$ protons on target(P.O.T.) is seen that may be due to damage. This would require the replacement of Si detectors once per a year for more high-intensity beam. At high intensity, more electron-ion pairs are created and distort the electric field inside the IC detector, which worsens the yield.
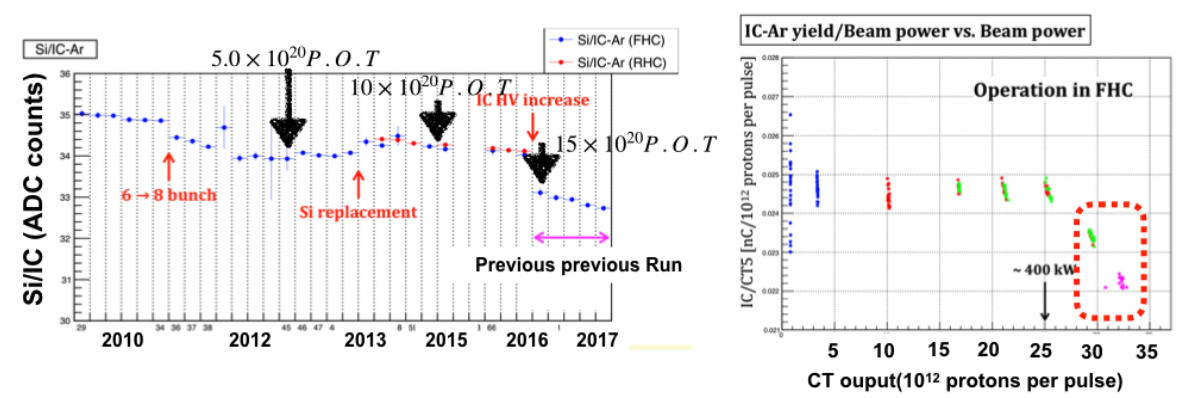

Figure 1: Degradation for Si detector (left) and worse signal response for IC at high intensity beam (right).

\section{Development of new detectors}

The J-PARC proton beam power is planned to be upgrade from $485 \mathrm{~kW}$ to $1.3 \mathrm{MW}$ in the future. The upgrade parameters are shown in Table $\mathrm{W}$. When upgraded, the radiation tolerance of MUMON will be more problematic, which leads a new MUMON development for the future J-PARC proton beam line. 


\begin{tabular}{|c|c|c|}
\hline & Now $(485 \mathrm{~kW})$ & Future plan $(1.3 \mathrm{MW})$ \\
\hline \hline \# of protons / pulse & $2.4 \times 10^{14}$ & $3.2 \times 10^{14}$ \\
\hline Operation cycle & $2.48 \mathrm{~s}$ & $1.16 \mathrm{~s}$ \\
\hline
\end{tabular}

Table 1: Current plan to increase the J-PARC proton beam power.

The most important performance requiring for the new detectors is high radiation-resistance. Diamond detectors, PMTs and EMTs (Electron Multiplier Tube) were tested. Diamond is expected to have high radiation hardness due to the high displacement energy compared to Si. An EMT has the same structure as a PMT except an EMT has no photocathode. The photocathode of EMT is replace to an aluminum foil, therefore EMTs are expected to have high radiation tolerance with a disadvantage of gain loss. Sb doping is applied to the dynodes of some of tested EMT, in order to recover gain loss.

\begin{tabular}{|c|c|c|c|}
\hline & A1, A2 & B1, B2 & C1, C2 \\
\hline \hline Edge & metaled & not metaled & metaled \\
\hline Dim. & $3.8 \times 3.8 \times 0.5\left(\mathrm{~mm}^{3}\right)$ & $4.0 \times 4.0 \times 0.5\left(\mathrm{~mm}^{3}\right)$ & $4.5 \times 4.5 \times 0.5\left(\mathrm{~mm}^{3}\right)$ \\
\hline Crystal & Element6 & Element6 & Element6 \\
\hline Electrode & Element6 & Element6 & CIVIDEC \\
\hline
\end{tabular}

Table 2: Properties of diamond detectors installed in J-PARC. A, B, C are the labels referring to the period the detectors were purchased ( $\mathrm{C}$ is the newest type). The columns for Crystal (diamond crystal) and Electrode are indicating manufacturing companies.

Six diamond detectors were installed in J-PARC neutrino beamline muon pit from 2012 - 2017 (Table [2). Diamond detectors have a $1 \%$ resolution for the muon intensity and linearity fluctuation of less than 5\%, which is almost comparable to the current detectors. However, the stability does not satisfy our requirement, which is a less than 3\% fluctuation(Figure [1). This means diamond detectors are not a good candidate for our experiment.

Two standard PMTs (Hamamatsu R9880) were installed and both the linearity and stability were checked. Unfortunately, at high-intensity beam, we found the PMTs to be unstable and have poor linearity (shown in Figure [3).

The detailed analysis of EMT data can be seen in the published paper[四]. EMTs having both dynodes with $\mathrm{Sb}$ doping and dynodes without Sb doping were tested. An original EMT was custom made with standard PMT (R9880). Another type of EMT, whose capacitances were modified, was tested in order to match the requirement of the J-PARC neutrino beam. The EMT time response, intensity resolution, and linearity were checked, which showed comparable or better performance than the current detectors. The EMT stability is shown in Figure 4 . A 3\% drift can be seen in the beginning of usage, however, the fluctuations are less than $1 \%$ after this initial drift. We conclude that EMTs are the best candidate for the future J-PARC neutrino beam muon monitor. 

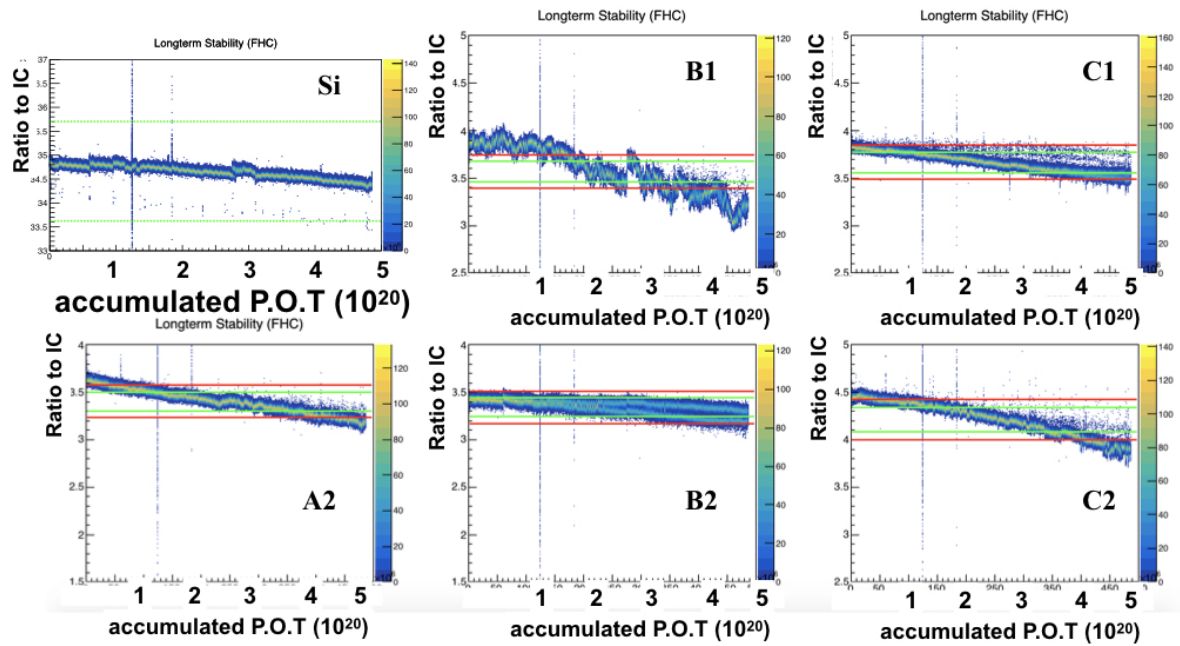

Figure 2: Stability of diamond detectors
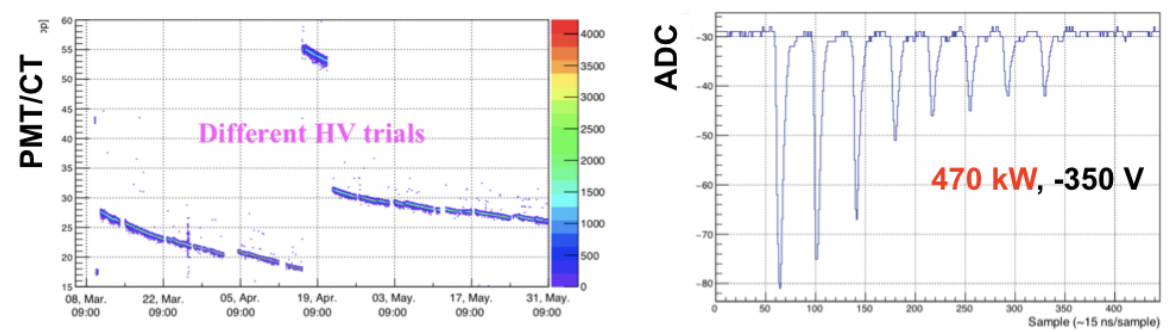

Figure 3: Stability (left) and raw waveform (right) of the PMT.
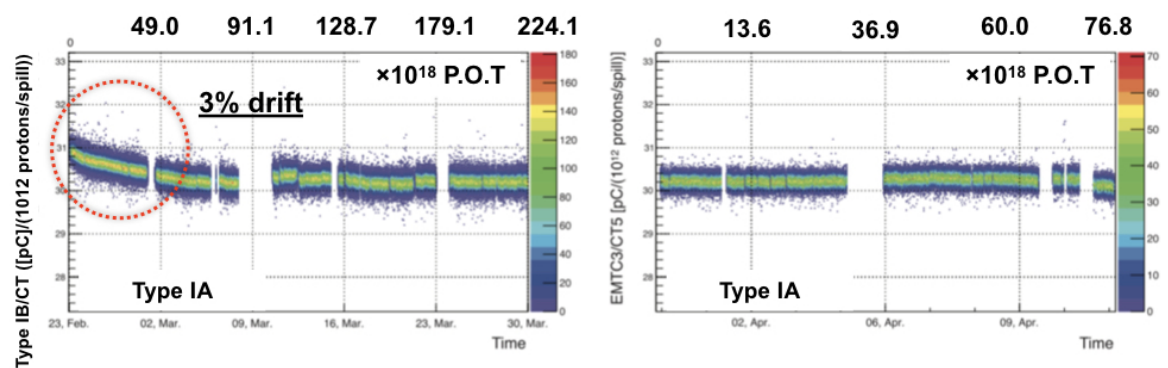

Figure 4: The left plot shows initial instability of EMT. Once EMT become stable, such klnd of behavior is not seen like right plot.

\section{EMT beam test}

EMTs are expected to have a better performance than the current detectors for the J-PARC neutrino beamline muon monitor. We have proposed a beam test for a more detailed investigation. 
The beam test will be conducted at the Research Center for Electron Photon Science (ELPH) in Tohoku University at the end of November 2019. We will test the EMT in terms of linearity and stability using an electron beam. Linearity will be checked from the current intensity $(1.7 \times$ $\left.10^{7} \mu / \mathrm{cm}^{2} / 3 \mu \mathrm{s}\right)$ to the future intensity $\left(3.9 \times 10^{7} \mu / \mathrm{cm}^{2} / 3 \mu \mathrm{s}\right)$. The electron exposure for the stability check corresponds to 10 years of muon beam exposure with the future J-PARC intensity $\left(2.9 \times 10^{15} \mu / \mathrm{cm}^{2}\right)$. The setup is shown in Figure [1.

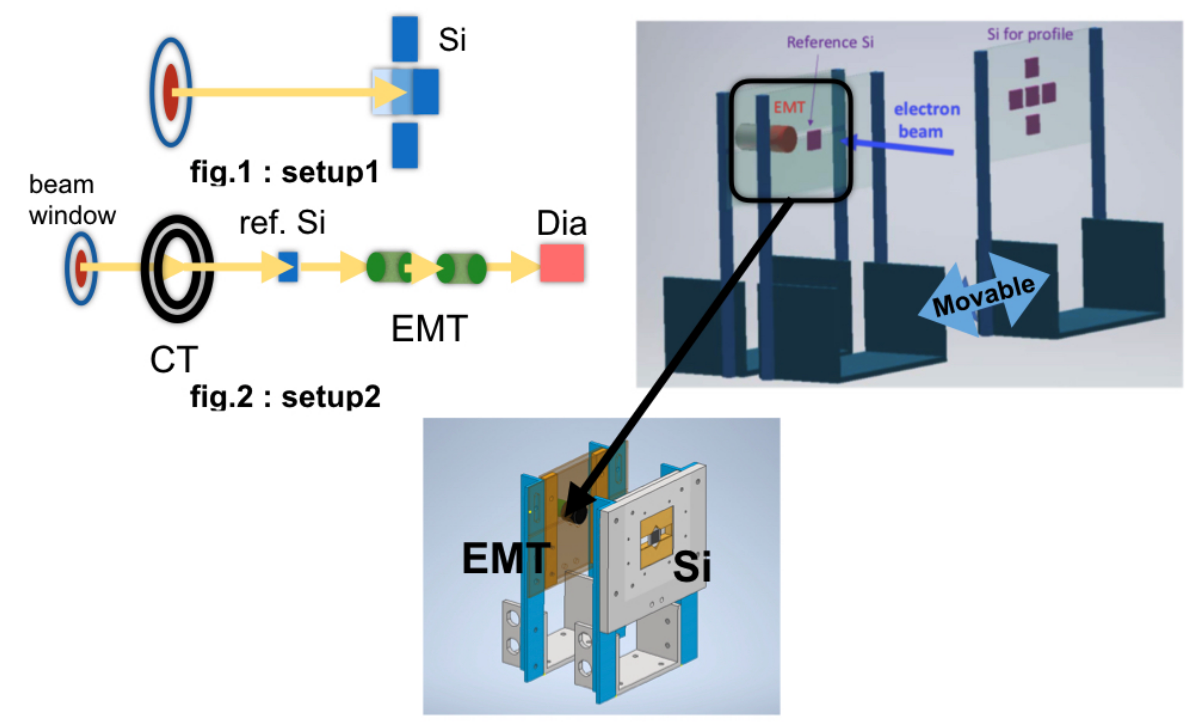

Figure 5: The current setup for the EMT beam test. CT is indicating "Current Transformer" to calibrate the beam current. Setup 1 is used for measuring the profile of the electron beam. A reference silicone sensor is installed for EMT performance cross-check.

\section{5. conclusion}

MUMON is essential for the T2K experiment to generate a properly oriented neutrino beam. The current MUMON detectors have some issues, which will be problematic for the future higher intensity beam of T2K. We are planning to develop new detectors for the MUMON. EMTs seem to have a better performance than current sensors based on preliminary tests, therefore a beam test will be conducted for a more detailed investigation.

\section{References}

[1] Y. Ashida et al., "A new electron-multiplier-tube-based beam monitor for muon monitoring at the T2K experiment ", Prog. Theor. Exp. Phys., 2018,10,103H01 (2018).

[2] K. Matsuoka et al., "Design and performance of the muon monitor for the T2K neutrino oscillation experiment", Nucl. Instrum. Meth. Phys. Res. A 624, 591 (2010). 